\title{
Identifying Important Action Primitives for High Level Activity Recognition
}

\author{
Atif Manzoor ${ }^{1}$, Claudia Villalonga ${ }^{2}$, Alberto Calatroni ${ }^{2}$, Hong-Linh Truong ${ }^{1}$, \\ Daniel Roggen ${ }^{2}$, Schahram Dustdar ${ }^{1}$, and Gerhard Tröster ${ }^{2}$ \\ 1 Distributed Systems Group, Vienna University of Technology, Vienna \\ \{manzoor, truong, dustdar\}ainfosys.tuwien.ac.at \\ 2 Wearable Computing Laboratory, ETH, Zürich \\ \{villalonga, alberto.calatroni, daniel.roggen, \\ troester\}aife.ee.ethz.ch
}

\begin{abstract}
Smart homes have a user centered design that makes human activity as the most important type of context to adapt the environment according to people's needs. Sensor systems that include a variety of ambient, vision based, and wearable sensors are used to collect and transmit data to reasoning algorithms to recognize human activities at different levels of abstraction. Despite various types of action primitives are extracted from sensor data and used with state of the art classification algorithms there is little understanding of how these action primitives affect high level activity recognition. In this paper we utilize action primitives that can be extracted from data collected by sensors worn on human body and embedded in different objects and environments to identify how various types of action primitives influence the performance of high level activity recognition systems. Our experiments showed that wearable sensors in combination with object sensors clearly play a crucial role in recognizing high level activities and it is indispensable to use wearable sensors in smart homes to improve the performance of activity recognition systems.
\end{abstract}

Keywords: Activity recognition, smart homes, action primitives.

\section{Introduction}

Smart homes aim to support people by adapting to their requirements to accomplish their goals and objectives in dynamically changing and continuously emerging situations. Human activity is the fundamental type of context to build many applications in such homes. Consequently, activity recognition has become an active research field to design dependable systems to recognize human activity. Different sensory modalities, including ambient sensors [136], vision based sensors [5]7, and wearable sensors [14], are used to observe the environment and reasoning algorithms work out sensor data to detect activities at different levels of abstraction, ranging from the basic short time low level human action primitives, such as sit, stand, and walk, to high level human activities of daily living (ADL) that span over comparatively longer periods of time, such as drinking coffee, eating sandwich, and cleaning room. Still one of the key challenges in building effective and reliable high level activity recognition systems is to identify an 
optimal set of primitives that express enough information to accurately recognize such human activities. Categorizing significant primitives will also decrease the overhead in terms of sensor cost, human effort, and computing resources. But there is yet a lack of research effort that needs to be undertaken to identify the impact of various types of action primitives in recognizing ADL. Most of the works have been limited to use action primitives from a single sensing modality to recognize ADL.

In this paper we identify the importance of the different types of action primitives, such as human locomotion and object usage primitives, to recognize ADL. Distinguishing the role of such primitives will be crucial for two reasons. First, it will help in designing an ambient intelligent environment to indicate where to place sensors, such as on body, in objects, or in the environment. Second, it will also indicate which action primitive are worthwhile to invest additional effort in designing action primitive spotting algorithms to recognize those action primitives. For example, "hand cutting the bread movement" is an important action primitive as it is giving a clear indication that subject is preparing a sandwich. We used the annotations of the EU project OPPORTUNITY data set [11] that are based on the recordings of the proceedings of data collection activity as action primitives. These annotations include body movement primitives, like walk, sit, and stand, arm movement primitives, like reach, release, and cut, and object or environment usage primitives, like use glass, move chair, and open fridge door. Our experiments determined that although human body locomotion primitives are rare to use in recognizing ADL, they showed better performance than object or environmental usage primitives in recognizing some of the ADL. Human body locomotion primitives used in combination with object and environmental usage primitives showed the best performance in recognizing ADLs that make using wearable sensors an indispensable choice to recognize ADL.

The reminder of the paper is organized as follows. Section 2 gives an overview of the existing work in literature. Section 3 briefly describes the process of activity recognition and classification algorithms that have been used in this work. Section 4 presents the detail of the data set that has been used in the experiments. Section 5 exhibits and discusses the result. Finally we present the conclusion of this research effort in Section 6 .

\section{Related Work}

Research efforts that had been undertaken to recognize ADL are mainly dominated by environmental change or the object motion / usage primitives. Kasteren et al. [13] recognized ADL by collecting and using environmental sensor data in a smart home. Sensors were embedded in doors, cupboards, and refrigerator. Mckeever et al. [8] also used the Van Kasteren data set [13]. Tapia et al. [12] used environmental change sensors that had been installed on doors, windows, cabinets, drawers etc. Lepri et al. [5] recognized the ongoing activities by using the visual sensors. They equipped the living room and kitchen of a flat with web cameras where different subjects performed activities of daily living. They processed the video streams to get the primitives about the location and posture of the subject to recognize high level activities. But these primitives had not proved to be enough to recognize activities like eating, drinking, and cleaning. Mostly aforementioned works used sensor systems that can provide primitives about the environmental state change. Lepri et al. [5] used human body posture primitives with their 
location to detect the ADL. Neither of these works used action primitives related to human body movements like walk, run, and reach.

Logan et al. [6] used the place lab, an instrumented home environment [4], to collect their data set. They used environment built-in sensors, object motion sensors, and RFID tags. Two 3-axis accelerometer sensors were also worn by subject on his limbs to show his motion. In their experiments they provided a comparison of accuracy that was achieved by environmental and object motion sensors. Their experiments showed that a combination of environmental and object motion sensors provided better results. But they only showed their results for all the activities collectively. They did not provide any information if a certain type of primitive provides better performance for a particular activity. As compared to their work we included action primitives extracted from wearable sensor data in our experiments. We also showed our results for each activity separately so we can distinguish which category of action primitives show better performance for a specific activity.

Maekawa et al. [7] used a customized sensor embedded with a camera, microphone, accelerometer, and a digital compass. That sensor set was worn as a wrist band and collect data while the subject was busy in performing different activities. Although they compared the performance of these sensors by using the data sets by including and excluding each type of sensor, they did not provide any comparison on the basis of different type of action primitives. As compared to these works we not only used the object motion / usage and environmental change action primitives but also included human locomotion primitives to classify ADL. Our experiments also showed a comparison of the accuracies of these action primitives, to recognize ADL, individually and in combination with each other.

\section{Activity Recognition from Action Primitives}

Common systems that recognize high level activities from different sensing modalities collect data from the sensors embedded in the environment and objects and worn by the human as shown in Figure 1. State of the art machine learning algorithms are used to classify action primitives from sensor data. These algorithms are first trained with sensor data to extract primitives of interest. Primitives give information about different current events in the environment, e.g., "subject is walking" describes human body motion. Sets of such primitives are provided to train machine learning algorithms to recognize activities that spread over a longer period of time. Many machine learning algorithms are available in WEKA [3] that provides researchers an easy access to stateof-the-art techniques in machine learning and had been used for activity recognition in different works such as [6]102].

We have used J48, Hidden naive Bayes (HNB), and IBK for the purpose of classification of high level activities. J48 is the WEKA implementation of C4.5 decision tree [9]. C4.5 decision tree is also used in [6 102] for activity recognition. C4.5 decision tree chooses one attribute of the data that most effectively splits its set of samples based on the criterion of normalized information gain (difference in entropy). The attribute with the highest normalized information determines the decision at each node. A Bayes classifier is a simple probabilistic classifier based on applying Bayes theorem 


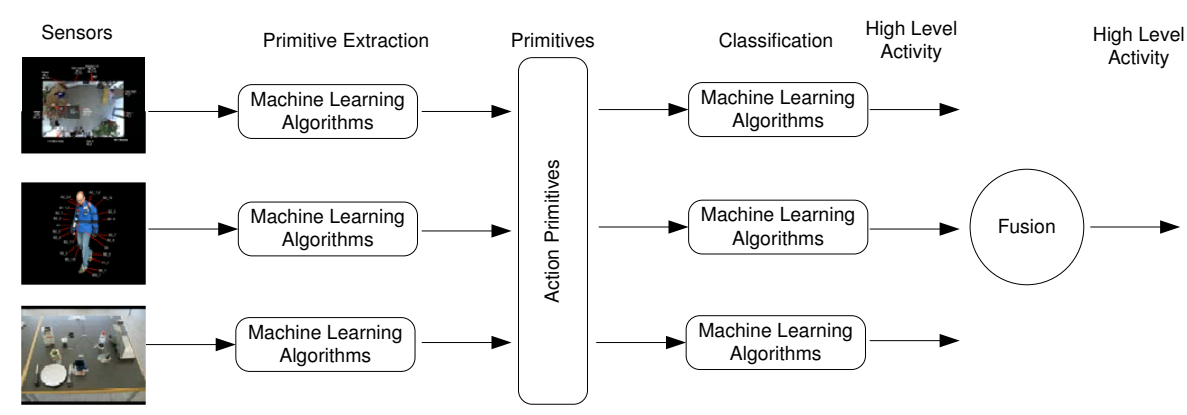

Fig. 1. Activity recognition process using different sensing modalities

with strong feature independence assumptions and is used in [6 10 2] for activity recognition. In this work we have used Hidden naive Bayes [15] that is an extended form of naive Bayes and accommodates the attribute dependencies. HNB creates a hidden parent for each attribute using the average of weighted one-dependence estimators. IBK [1] implements $k$-nearest neighbor, an instance-based learning algorithm that generates classification prediction using only specific instances. $k$-nearest neighbor is also used in [102] for activity recognition. We have chosen these commonly used classification algorithms considering different learning strategies used in these algorithms, such as decision tree, probability based learning, and instance based learning. We did not build the histograms of sensor data over a window of time and classified all the samples collected at an instance of time. Reason for these choices was to put more emphasis on the study of the influence of primitive actions on activity recognition rather than on the powers of more advanced classification algorithms or strategies.

A person performs different activities in different parts of a house. She may be busy in kitchen while preparing breakfast or she may be relaxing in the lounge. The different nature of these activities implies that these activities are composed of different primitives. The composition of these activities require to look at each activity individually that will not only give us the opportunity to observe which type of sensors should be used to recognize which activity but it will also indicate which type of sensors should be used in which part of house. Considering these requirements first we look at the influence of each activity individually. Then we study the influence of different action primitives collectively.

\section{Data Description}

We used the data sets that have been collected in the EU project OPPORTUNITY [11]. The data set about the naturalistic human activities was collected in a sensor rich environment: a room simulating a studio flat with kitchen, deckchair, and outdoor access where subjects performed daily morning activities. 15 networked sensor systems with 72 sensors of 10 modalities were deployed integrated in the environment, objects, on the body. The deployment of the large number of networked sensor systems of different modality make this data set ideal to study the impact of different sensing modalities 
in activity recognition. Table 1 shows a short description of those activities and their duration for a single run. Twelve subjects executed activities of daily living in this environment, yielding an average of 2 hours of effective data per subject, for a total twenty five hours of sensor data. According to our estimations over 11000 primitives of interactions with objects and over 17000 primitives of interactions with environment have been recorded. This makes the data set highly rich in gesture primitives and the largest for the purpose of multimodal activity recognition.

Table 1. Different activities and duration for single run (in seconds)

\begin{tabular}{|l|l|l|}
\hline Activities & Description & Duration $(s)$ \\
\hline \hline Idle & Not performing any activity & 583 \\
\hline Relaxing & Go outside and have a walk & 157 \\
\hline Early morning & Move around in the room and casually check the objects & 276 \\
\hline Coffee time & Prepare coffee with milk and sugar using coffee machine and drink it & 129 \\
\hline Sandwich time & $\begin{array}{l}\text { Prepare sandwich with bread, cheese, and salami using bread cutter, various knives, } \\
\text { and plates and eat it }\end{array}$ & 375 \\
\hline Clean up & Put objects used to original place or dish washer and cleanup the table & 183 \\
\hline
\end{tabular}

Table 2 shows the different action primitives that are used in our experiments. These action primitives are extracted from the annotations of the data set performed by experts using the videos of all the proceedings during data collection process. The experts identified all the actions performed by the subject during his activities. Table 3 shows all the sensors that have been deployed in the environment and the type of action primitives that can be extracted from these sensors. Locomotion primitives can be extracted from data collected by the sensors worn on the subject body and include action primitives such as walking, sitting, and lying. Arm locomotion primitives can be extracted from data collected by the sensors worn on the arms of the subject and include the action primitives such as cut, spread, and release. The object data is collected from the interaction of sensors embedded in the arms and objets. Primitives extracted from this data present whether a particular object is used at a specific instance of time. Multiple sensors of different modalities made this data set ideal to perform activity recognition in an opportunistic environment and observe the effectiveness of different sensing modalities. We have used the data of five runs of a single subject.

Table 2. Brief description and values of action primitive categories

\begin{tabular}{|c|c|c|}
\hline $\begin{array}{|ll|}\begin{array}{l}\text { Action } \\
\text { category }\end{array} & \text { primitive } \\
\end{array}$ & Description & Primitive values \\
\hline Locomotion & basic human movements & walk, run, stand, lie, sit, stairs up, stairs down \\
\hline Left arm locomotion & left arm movements & \multirow{2}{*}{$\begin{array}{l}\text { reach, move, release, lock, unlock, open, close, stir, sip, bite, } \\
\text { clean, cut, spread }\end{array}$} \\
\hline Right arm locomotion & right arm movements & \\
\hline Left arm object & left hand interaction with objects & \multirow{2}{*}{$\begin{array}{l}\text { fridge, dishwasher, drawer1 (top), drawer2 (middle), drawer3 } \\
\text { (lower), door1, door2, switch, table, cup, chair, glass, spoon, } \\
\text { sugar, knife salami, knife cheese,salami, bottle, plate, cheese, } \\
\text { bread, milk, lazy chair }\end{array}$} \\
\hline Right arm object & right hand interaction with objects & \\
\hline
\end{tabular}


Table 3. Sensor systems locations and observations

\begin{tabular}{|l|l|}
\hline Sensor system & Location and observation \\
\hline \hline Commercial wireless microphone & Chest and dominant wrist. Senses user activity \\
\hline Custom wireless Bluetooth acceleration sensors & 12 locations on the body. Senses limb movement \\
\hline Custom motion jacket & $\begin{array}{l}\text { Jacket including 5 commercial RS485-networked XSense inertial mea- } \\
\text { surement units }\end{array}$ \\
\hline Custom magnetic relative positioning sensor & $\begin{array}{l}\text { Emitter on shoulder, receiver on dominant wrist. Senses distance of hand } \\
\text { to body }\end{array}$ \\
\hline Commercial InertiaCube3 inertial sensor system & One per foot, on the shoe toe box. Senses modes of locomotion \\
\hline Commercial Sun SPOT acceleration sensors & One per foot, right below the outer ankle. Senses modes of locomotion \\
\hline $\begin{array}{l}\text { Custom wireless Bluetooth acceleration and rate } \\
\text { of turn sensors }\end{array}$ & On 12 objects used in the scenario. Senses object use \\
\hline Commercial wired microphone array & 4 at one room side. Senses ambient sound \\
\hline Commercial Ubisense localization system & Corners of the room. Senses user location \\
\hline Axis network cameras & 3 locations, for localization, documentation and visual annotation \\
\hline XSense inertial sensor & On the table and chair. Senses vibration and use \\
\hline USB networked acceleration sensors & USB networked acceleration sensors \\
\hline Reed switches & 13 , on doors, drawers, shelves. Sense usage, provides ground truth \\
\hline Custom power sensors & Connected to coffee machine and bread cutter. Senses usage \\
\hline Custom pressure sensors & 3 on the table, user placed plates and cups on them. Senses usage \\
\hline
\end{tabular}

\section{Experiments}

In our experiments we analyzed the impact of the different combinations of action primitives on high level activity prediction. For this purpose we divided the different types of action primitives in seven different combinations. First, we conducted experiments for every individual activity with all primitive sets. Later, we also observed the impact of primitive sets considering all activities collectively. In this section we will discuss the different primitive sets, the impact of those sets on each activity individually and all activities collectively, and finally we will discuss the results and present our recommendations.

\subsection{Primitive Sets}

Table 4 shows the action primitive sets and the categories of action primitives that have been used in those sets. In S1 we used all the action primitive categories described in Table 2. In S2 we excluded the left arm object movement action primitives and the right arm object movement action primitives. In this set we depended upon the action primitives extracted from wearable sensors like arm motion, e.g., moving, reaching, releasing an object. In S3 we also excluded all the wearable sensors that give us information about the locomotion of human limbs. In this set we used only with action primitives that have values about human actions like walk, sit, and stand. In S4 we used action primitives with both arms locomotion. In S5 and S6 we used left and right arm locomotion respectively. In S7 we used only object sensors, i.e., we had only information about the use of a specific object and we did not have any information that whether concerned person was sitting, standing, lying, or walking. Similarly we did not have any information which hand is used to handle an object. We used these different combination of sensors with the classification algorithms discussed in Section 3 . Table 1 presents the detail of the values of these primitives. 
Table 4. Different sets of action primitives

\begin{tabular}{|l|l|}
\hline Action primitive set & Categories of action primitives \\
\hline \hline$S_{1}$ & locomotion, left arm movements, right arm movements, left arm object, right arm object \\
\hline$S_{2}$ & locomotion, left arm movement, right arm movement \\
\hline$S_{3}$ & locomotion \\
\hline$S_{4}$ & left arm movement, right arm movement \\
\hline$S_{5}$ & right arm movement \\
\hline$S_{6}$ & left arm movement \\
\hline$S_{7}$ & object movement \\
\hline
\end{tabular}

\subsection{Action Primitives Impact in Recognizing Each Activity Individually}

Here we analyze the impact of these primitive sets in recognizing each activity individually. Figure 2 shows the true positive rate of the classification algorithms J48, HNB, and IBK using all primitive sets to recognize the activity Idle. The classifiers showed particularly better performance using the action primitive sets S4, S5, S6, and S7. The action primitive sets S4, S5, and S6 present the action primitives extracted from the wearable sensors worn on the limbs. These primitives include information whether the subject has used an object or not. The action primitive set S7 consists of the primitives extracted from object sensors. This action primitive set also provides information about the usage of objects available in the environment. Although primitive sets extracted from object sensors proved relatively better, primitive sets that have been extracted from other sensory modalities are also close. This result was not surprising as when the subject is idle, she is neither interacting with any of the objects nor making much movements. So action primitive extracted from wearable sensor or the objet sensors are not particularly crucial for recognizing this activity and any sensory modality can easily detect whether the subject is idle or not.

Figure 3 shows the true positive rate of the classification algorithms J48, HNB, and IBK using all action primitive sets to recognize the activity Relaxing. This activity proved to be most difficult one to recognize. During this activity the subject was either

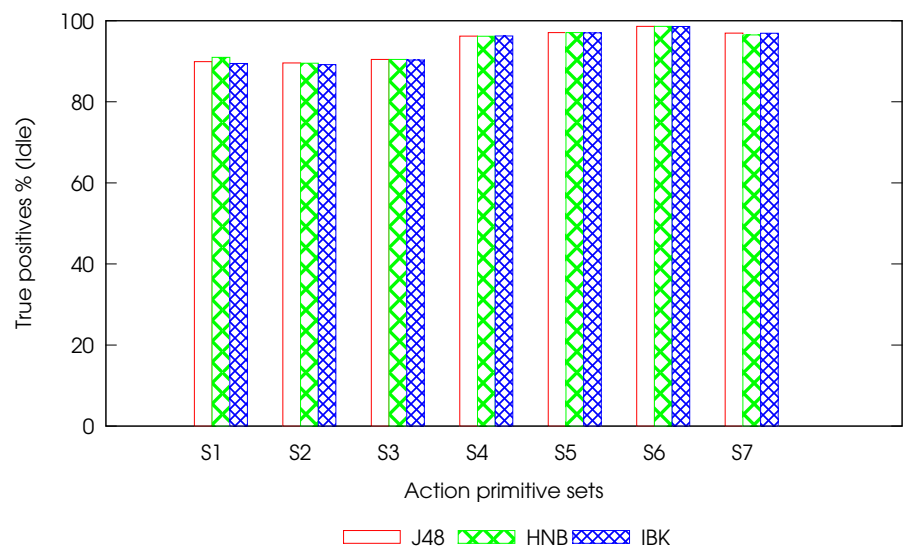

Fig. 2. True positive percentage of activity Idle 


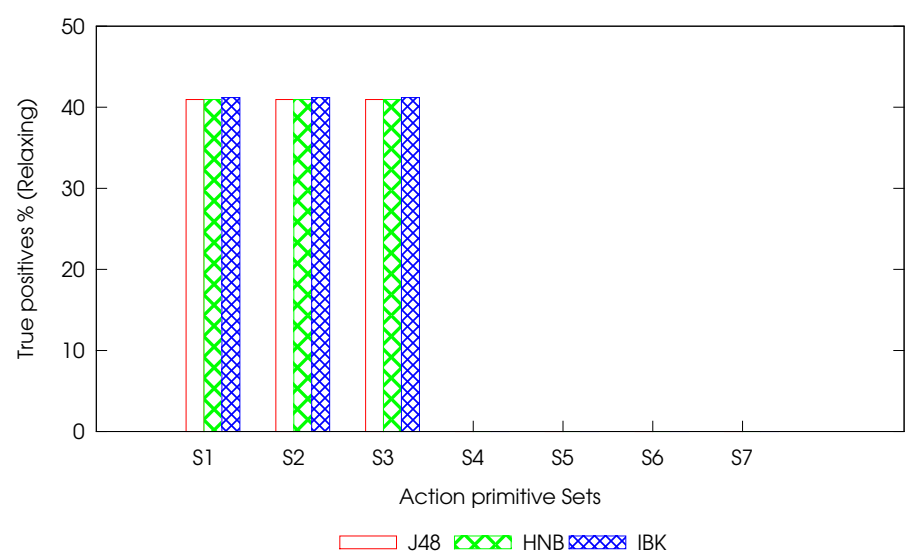

Fig. 3. True positive percentage of activity Relaxing

taking rest or was casually moving around the building. She was neither particularly involved in any activity nor interacting with any object in the environment. The true positive rate to recognize this activity indicates that the classifiers completely failed to recognize this activity using the action primitive sets S4, S5, S6, and S7. The Main reason was that during this activity the subject was neither performing any physical movements nor interacting with the environment and the objects. Classifiers get almost the same feature for this activity as the Idle activity. Comparatively the high number of idle activity overwhelmed the decision of classifiers and they got completely confused to distinguish between Idle and Relaxing activities. The classifiers detected almost all of the Relaxing activities as the Idle activity. This is also evident from the high false positive rate of J48 using primitive sets S4, S5, S6, and S7 as shown in Figure 8 Primitive sets S1, S2, and S3 proved better in recognizing this activity as the subject was comparatively more dynamic than being completely Idle. Recognizing Relaxing activity becomes impossible when primitive sets exclude primitives extracted from wearable locomotion sensors. Consequently human body locomotion action primitives extracted from wearable sensors proved vital in recognizing this activity.

Figure 4 shows the true positive rate of classification algorithms J48, HNB, and IBK using all action primitive sets to recognize the activity Early_morning. During this activity the subject moved in the room, and randomly checked some objects in the drawers and on the shelf. Although the classifiers using primitive sets S4, S5, and S7 showed better performance in recognizing this activity as compared to recognizing Relaxing activity, wearable sensors providing locomotion primitives proved better in this case too. The main reason was that during this activity the subject spends a lot of time to perform physical activities. Again in this case she did not interact much with objects available in the environment. Resultantly, action primitives extracted from object sensors were not able to recognize this activity. Left hand locomotion action primitives proved useless in recognizing this activity. The main reason for their failure is that the subject was casually interacting with different objects and only used dominant right hand for this purpose. Wearable sensor providing human locomotion primitives again proved vital in this case. 


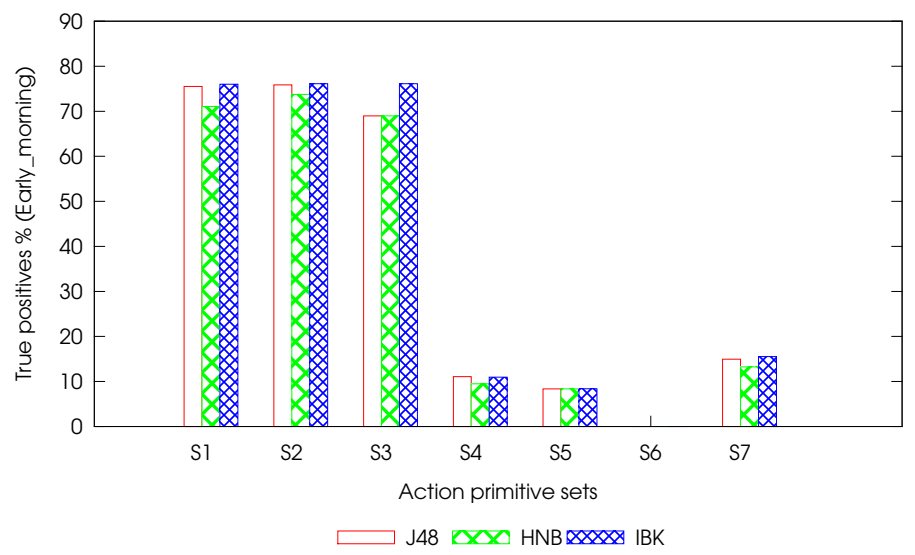

Fig. 4. True positive percentage of activity Early_morning

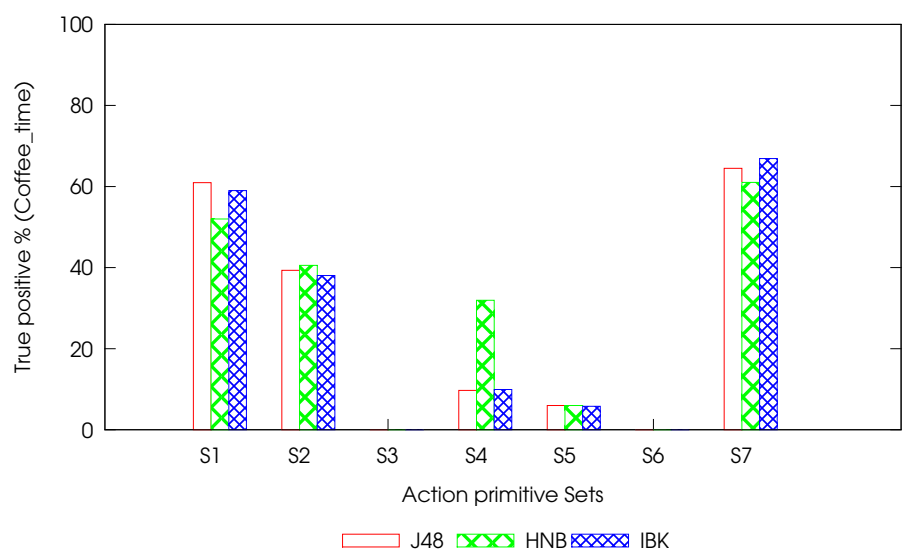

Fig. 5. True positive percentage of activity Coffee_time

Figure 5 shows the true positive rate of the classifiers using all primitive sets to recognize the activity Coffee_time. During this activity the subject prepared coffee with milk and sugar by using a machine, took sips of coffee and also interacted with different objects in the environment. As evident from the activity description, this activity is more distinctive on the basis of objects that were used during this activity than human locomotion action primitives. Subsequently object usage primitives also performed comparatively better than human body motion primitives in recognizing this activity.

Figure 6 shows the true positive rate of the classification algorithms J48, HNB, and IBK using all primitive sets to recognize the activity Sandwich_time. During this activity the subject interacted with different objects in the environment like bread, cheese, and salami, and bread cutters to prepare the sandwiches. Later the subject ate the sandwiches. Contrasting to Idle activity when the subject was motionless most of the time and interacted with few objects, in this activity the subject not only performed many 


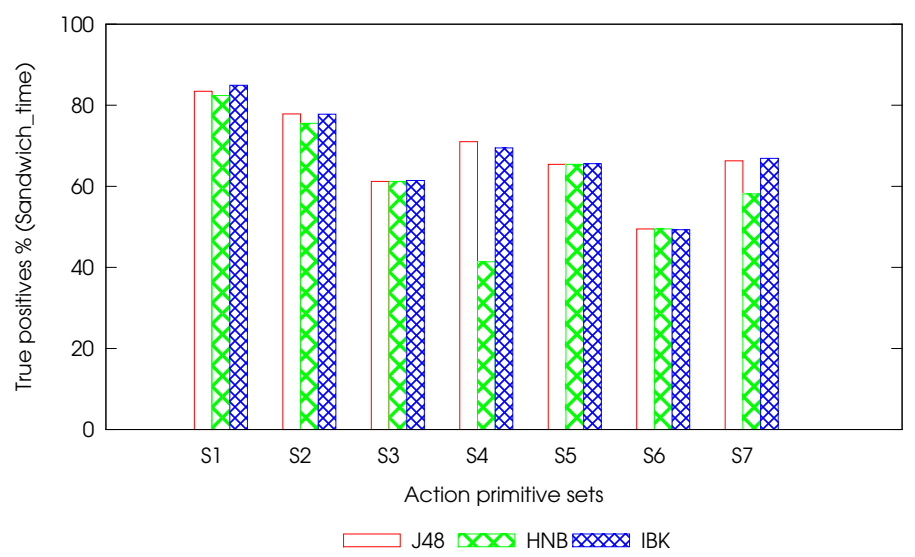

Fig. 6. True positive percentage of activity Sandwich_time

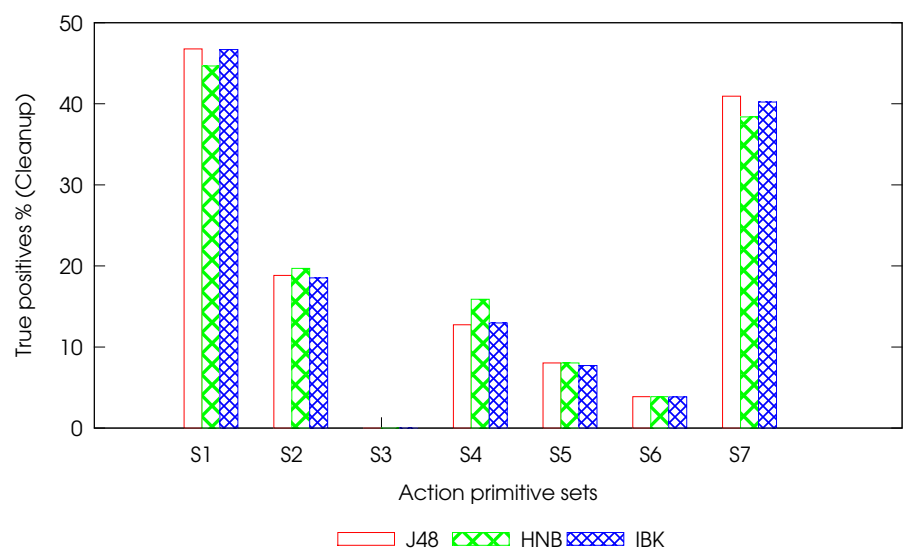

Fig. 7. True positive percentage of activity Cleanup

low level physical activities like cutting the bread but has also interacted with various objects in the environment. As a result all primitive sets proved comparatively better in recognizing this activity. Human body action primitives that can be extracted from wearable sensors data provide better rate of true positives as compared to object usage primitives. But in case of this activity the classifiers clearly performed better when the reason with primitive set that used combination of all action primitives extracted from wearable sensors and object sensors. Combining the action primitives from wearable sensors with primitives about the usage of objects available in the environment provided a clear evidence about Sandwich_time activity as indicated by the high true positive rate of algorithms using sensor set S1 in Figure 6

Figure 7 shows the true positive rate of the classifiers using all primitive sets to recognize the activity Cleanup. This activity was the final activity in the drill run for data collection. During this activity the subject put all objects used to original places or dish 
washer and cleanup the table. Classification algorithms could not show good accuracy for this activity. Body locomotion primitives, such as walk, sit, and stand, proved inadequate when the classifiers used these primitives alone to detect Cleanup activity. However, limbs locomotion primitives, such as reach, move, and release, proved better in recognizing this activity. If we compare the performance of all the action primitive sets when used individually, the object usage primitive set showed the best performance as shown in Figure 7 Overall classifiers showed best performance while reasoning with the primitive sets that used combination of human locomotion primitives, limbs locomotion primitives, and object usage primitives to detect Cleanup activity.

\subsection{Action Primitives Impact in Recognizing All Activities}

Figure 8 shows the weighted averages of true positive rates, false positive rates, precision, recall, f-measure, and roc area of $\mathrm{J} 48$ classification algorithms while recognizing all activities. The classifier shows highest value of true positive rate with lowest value of false positive rate while using the action primitive set $\mathrm{S} 1$ that includes all available types of action. Subsequently we also have good values of other metrics for primitive set $\mathrm{S} 1$. Although the use of wearable sensors to recognize high level activities is very rare the classifier also showed comparatively good performance while using primitive set S2 that only contains primitives extracted from wearable sensor data. The reason for the good performance of the classifier using primitive set S2 is the comprehensive nature of the action primitives that were extracted from wearable sensor data. S2 not only provide information about the action primitives like walk, sit, and stand but also indicate that one of the objects available in the environment is used. These primitives also proved very helpful in recognizing activities like Idle, when subject is not

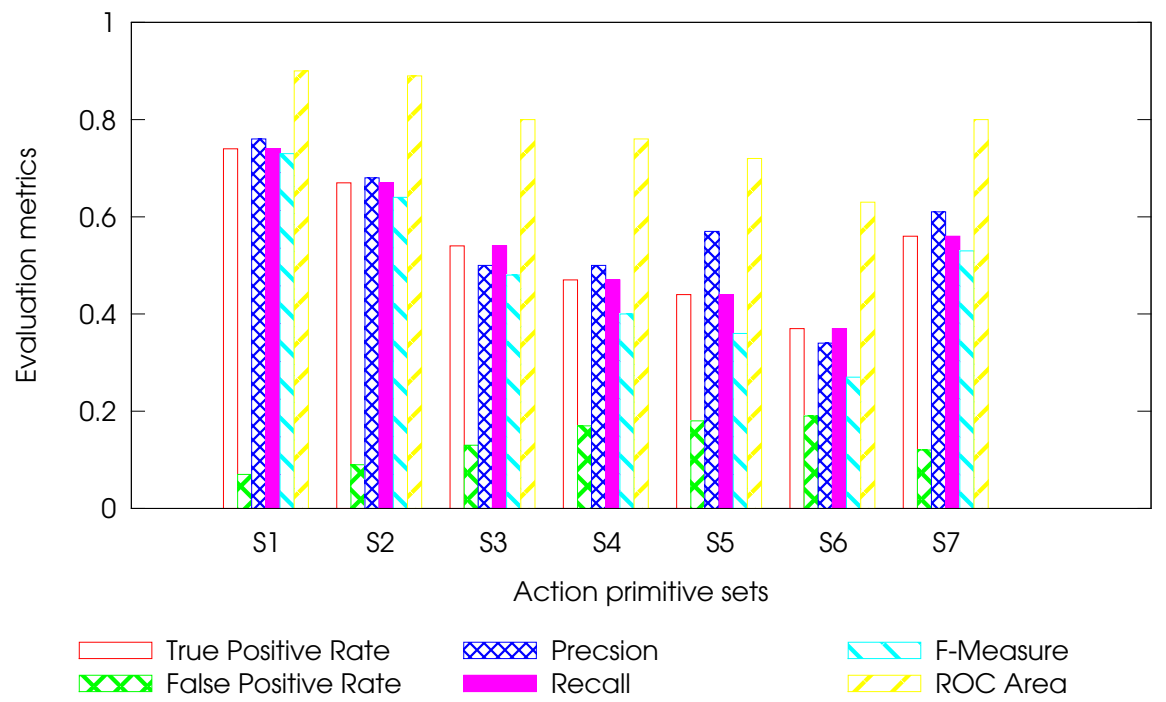

Fig. 8. Evaluation metrics for different primitive sets using decision tree 
performing any activity, Early_morning, when subject is walking around and handling different objects, and Relaxing when subject is siting or lying. However, classifiers get confused when they have to detect a single activity among ones that used same objects as there have not been any information about which object is used as shown by the high value of false positive rate and low value of precision as compared with sensor set S1.

The Classifier also showed comparable performance while using primitive set S3 and primitive set S7. While reasoning with S3 and S7, weighted averages of different evaluation metrics of J48 classifier to recognize all activities are almost equal as shown in Figure 8. The main reason is that human locomotion primitives are better in recognizing activities like Relaxing and Early_morning while object sensors were proved better in detecting activities in which subject have higher number of interaction with different objects such as Sanwich_time. The classifier, using these sets, got confused in recognizing other activities as evident by their high false positive rate and low value of precision. Classifier, using primitive set S4, S4 has not performed as good as while using primitive set S3 and S7. Although the classifiers reasoning with both of primitive sets S5 and S6 showed good performance for some of the activities, they could not show overall good accuracy. Primitive set S7 proved comparatively better. Clearly primitive set S1 that used combination of object sensors with wearable sensors proved the best to recognize human activities in smart home environment.

\subsection{Discussion and Recommendations}

Figure 8 shows that the classifiers have nearly equal values of the weighted average of true positive rates of recognizing all activities while using human locomotion action primitives and object usage action primitives individually. But if we consider each activity separately that is detected by the classifiers while using different action primitive sets as shown in Figures 2 - 7, we find that those activities are completely different for each type of action primitives. While using human locomotion action primitives individually, the classifiers performed better in recognizing those activities where the subject has less interaction with objects available in the environment. These activities include Idle, Relaxing and Early_morning. The classifiers completely failed in recognizing these activities while using objects and environment usage action primitives alone. But while using object and environmental usage action primitives classifiers performed better in recognizing activities that involve a lot of interaction with objects and environment. These activities include Coffee_time, Sandwich_time, and Cleanup. Collectively the classifiers performed best while using object and environmental usage action primitives and human body locomotion action primitives together.

Limbs locomotion primitives, like reach, cut, and touch, also proved significant in recognizing those activities that include not only using but also performing actions on different objects. Classifiers performed better while working with limbs locomotion primitives extracted from wearable sensors attached with dominant limb than the other limb. Wearable sensors that can be used to extract action primitives like sip or bite are also important in correctly distinguishing activities like drinking coffee or eating a sandwich. As action primitives extracted from wearable sensors used in combination with action primitives extracted from ambient sensors clearly proved better in recognizing high level human activities than action primitives extracted from ambient sensors 
alone, it is indispensable to use wearable sensors in smart environments to improve the performance of the classifiers to recognize high level human activities. We highly recommend to use wearable sensors with dominant limbs to extract locomotion action primitives. We found that object usage primitives are quite fundamental in recognizing the activities that were performed in areas where the subject have more interaction with the environment and objects, such as home kitchen. It is very important to install ambient sensors in these areas. Limbs locomotion primitives are also proved helpful in recognizing kitchen area activities.

\section{Summary}

In this paper we compare the performance of the classifiers reasoning with different sets of action primitives to recognize high level human activities. The main purpose of this study is to analyze the impact of the diverse type of action primitives on high level activity recognition and identify important action primitives. We include human body and limbs locomotion action primitives along with objects and environment usage action primitives in our experiments. While reasoning with human body locomotion action primitives individually, the classifiers show good performance in recognizing activities that do not involve interaction with environment, such as Idle, Relaxing, and Early_morning activities. The classifiers failed to recognize these activities while working with objects and environment usage action primitives. This fact makes wearable sensors that can be used to extract human body locomotion primitives an indispensable choice in the development of human activity recognition system. Overall the classifiers show best performance while using human body locomotion action primitives with objects and environmental usage action primitives. The classifiers also show better performance while using limbs locomotion primitives related to dominant arm than using limbs locomotion primitives related to other arm. We recommend to use wearable sensors with dominant limbs and ambient sensors at places where a person have a lot of interaction with environment and objects, like home kitchen, to effectively recognize high level human activities.

Acknowledgements. This work is financially supported by EU FP7 STREP Project SM4ALL (Smart hoMes for ALL) under Grant No. 224332 and EU FP7 FET Project OPPORTUNITY under Grant No. 225938.

\section{References}

1. Aha, D., Kibler, D.: Instance-based learning algorithms. Machine Learning 6, 37-66 (1991)

2. Bao, L., Intille, S.S.: Activity recognition from user-annotated acceleration data. In: Proceedings of Internation Conference of Pervasive Computing 2004, pp. 1-17 (2004)

3. Hall, M., Frank, E., Holmes, G., Pfahringer, B., Reutemann, P., Witten, I.H.: The weka data mining software: an update. SIGKDD Explor. Newsl. 11(1), 10-18 (2009)

4. Intille, S., Larson, K., Tapia, E., Beaudin, J., Kaushik, P., Nawyn, J., Rockinson, R.: Using a live-in laboratory for ubiquitous computing research. In: Fishkin, K.P., Schiele, B., Nixon, P., Quigley, A. (eds.) PERVASIVE 2006. LNCS, vol. 3968, pp. 349-365. Springer, Heidelberg (2006) 
5. Lepri, B., Mana, N., Cappelletti, A., Pianesi, F., Zancanaro, M.: What is happening now? detection of activities of daily living from simple visual features. In: Personal and Ubiquitous Computing, pp. 1-18 (2010)

6. Logan, B., Healey, J., Philipose, M., Tapia, E.M., Intille, S.: A long-term evaluation of sensing modalities for activity recognition. In: Krumm, J., Abowd, G.D., Seneviratne, A., Strang, T. (eds.) UbiComp 2007. LNCS, vol. 4717, pp. 483-500. Springer, Heidelberg (2007)

7. Maekawa, T., Yanagisawa, Y., Kishino, Y., Ishiguro, K., Kamei, K., Sakurai, Y., Okadome, T.: Object-based activity recognition with heterogeneous sensors on wrist. In: Floréen, P., Krüger, A., Spasojevic, M. (eds.) Pervasive Computing. LNCS, vol. 6030, pp. 246-264. Springer, Heidelberg (2010)

8. McKeever, S., Ye, J., Coyle, L., Dobson, S.: Using dempster-shafer theory of evidence for situation inference. In: Barnaghi, P., Moessner, K., Presser, M., Meissner, S. (eds.) EuroSSC 2009. LNCS, vol. 5741, pp. 149-162. Springer, Heidelberg (2009)

9. Quinlan, R.: C4.5: Programs for Machine Learning. Morgan Kaufmann Publishers, San Mateo (1993)

10. Ravi, N., Dandekar, N., Mysore, P., Littman, M.L.: Activity recognition from accelerometer data. American Association for Artificial Intelligence (2005)

11. Roggen, D., Calatroni, A., Rossi, M., Holleczek, T., Förster, K., Tröster, G., Lukowicz, P., Bannach, D., Pirkl, G., Ferscha, A., Doppler, J., Holzmann, C., Kurz, M., Holl, G., Chavarriaga, R., Creatura, M., del Millàn, R.: Collecting complex activity data sets in highly rich networked sensor environments. In: Proceedings of the 7th International Conference on Networked Sensing Systems, INSS 2010. IEEE, Los Alamitos (2010)

12. Tapia, E.M., Intille, S.S., Larson, K.: Activity recognition in the home using simple and ubiquitous sensors. In: Ferscha, A., Mattern, F. (eds.) PERVASIVE 2004. LNCS, vol. 3001, pp. 158-175. Springer, Heidelberg (2004)

13. van Kasteren, T., Noulas, A., Englebienne, G., Kröse, B.: Accurate activity recognition in a home setting. In: Proceedings of the 10th International Conference on Ubiquitous Computing, UbiComp 2008, pp. 1-9. ACM, New York (2008)

14. Zappi, P., Lombriser, C., Stiefmeier, T., Farella, E., Roggen, D., Benini, L., Tröster, G.: Activity recognition from on-body sensors: accuracy-power trade-off by dynamic sensor selection. In: Verdone, R. (ed.) EWSN 2008. LNCS, vol. 4913, pp. 17-33. Springer, Heidelberg (2008)

15. Zhang, H., Jiang, L., Su, J.: Hidden naive bayes. In: Twentieth National Conference on Artificial Intelligence, pp. 919-924. AAAI Press, Menlo Park (2005) 